\title{
Place de la prostaglandine E1 intra urétrale dans le traitement de l'impuissance érectile
}

\author{
E. AMAR
}

17 avenue Victor Hugo, 75016 Paris

\section{RESUME}

La place de l'asprostadil intra-urétral (Muse) dans le traitement de l'insuffisance érectile reste maintenant à définir depuis l'avènement des thérapeutiques per os et en particulier bien sûr le Sildenafil. L'alprostadil intra-urétral est un traitement local efficace de l'insuffisance érectile dans environ 35 à 40 $\%$ des cas. Les études comparatives avec les injections intra-caverneuses et le sildenafil sont en faveur de ces derniers mais le Muse conserve des indications et doit rester une arme dans l'arsenal thérapeutique en particulier dans les contre-indications du sildenafil et les cas de refus des injections intracaverneuses.

Mots-Clés : Insuffisance érectile; Alprostadil intraurétral ; Prostaglandine E1 ; injections intra-caverneuses ; Sildenafil ; Etude comparative.

\section{INTRODUCTION}

La PROSTAGLANDINE E1 sous forme d'ALPROSTADIL a été proposée en 1986 par ADAIKAN et IsHII [1-7] pour le traitement par injections intra-caverneuses. En 1994, PadmaNathan et collègues ont pour la première fois introduit l'application intra-urétrale sous forme de MUSE [10] (médicated urethral system for erection). Ce produit fut commercialisé en décembre 1996 aux USA et a connu une popularité grandissante jusqu'à ce que deux facteurs interviennent : d'une part les résultats un peu décevants de la pratique quotidienne et d'autre part la sortie du Sildenafil (VIAGRA*) en mai 1998 aux USA et en septembre 1998 en Europe.

\section{Nous allons donc}

1) faire un rappel sur l'action de la PGE1

2) Etudier l'originalité de la voie intra-urétrale.

3) Rapporter les résultats de l'étude américaine et européenne d'investigation à 3 mois et à un an.

4) Etudier les résultats critiques publiés dans la littérature.

5) Expliquer les possibilités éventuelles d'amélioration du système MUSE.

6) Rapporter les quelques études comparatives avec le traitement par injections intracaverneuses et par le Sildenafil.

7) Dégager la place actuelle et future du système MUSE dans le traitement de l'insuffisance érectile. 


\section{ACTION PHARMACOLOQUE DE LA PGE1 (ALPROSTADIL)}

\section{4 mécanismes sont mis en jeu :}

1) Stimulation de l'Adénylate Cyclase avec accumulation intra-cellulaire de l'AMP cyclique et aussi diminution du Calcium intra-cellulaire.

2) Stimulation directe des maxi canaux potassiques entraînant une hyper-polarisation.

3) Inhibition de la sécrétion d'adrénaline par l'intermédiaire des récepteurs pré-synaptiques des alpha 1 adréno-récepteurs, entraînant une diminution du tonus adrénergique.

4) Suppression de secrétion d'Angiotensine II.

Chacun de ces différents effets pharmacologiques contribue à la relaxation du muscle lisse caverneux.

\section{LA VOIE INTRA-URETRALE (MUSE)}

\section{A. PHARMACOLOGIE}

1. Le MUSE est résorbé rapidement et de façon efficace au niveau de l'urètre de l'homme : $80 \%$ d'une dose de $1000 \mathrm{mcg}$ sont résorbés au bout de 100 minutes.

2. Les pics plasmatiques de PGE1 se situent autour de 11,4 picogrammes par millilitre après l'administration de $1000 \mathrm{mcg}$ de MUSE, taux très faible à la limite de la détection dans le sang périphérique.

3. Dans l'éjaculat, l'augmentation moyenne de la quantité totale de PGE1 est de $123 \mathrm{mcg}$ en moyenne après administration de 1000 $\mathrm{mcg}$, soit une augmentation inférieure à la variabilité normale intra-individuelle des taux de prostaglandine totale [6].

4. Absence d'incidence in vitro sur la mobilité, la vitalité et la structure de la membrane des spermatozoïdes.

\section{B. PRESENTATION}

Le système se présente sous forme d'une canule prête à l'emploi stérile, avec un poussoir d'activation aisée avec deux doigts, l'alprostadil étant sous la forme d'un petit implant de cire solide de $125 \mathrm{mcg}, 250 \mathrm{mcg}, 500 \mathrm{mcg}$ ou $1000 \mathrm{mcg}$ (cf photo).

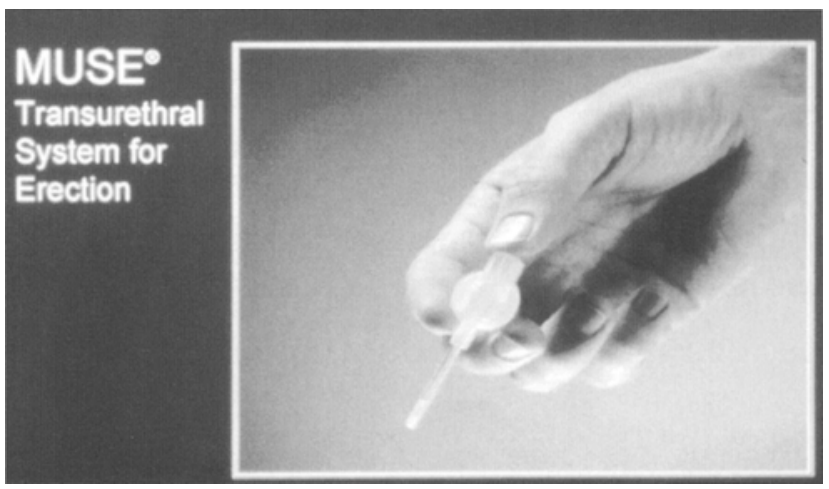

Le produit doit être conservé entre 4 et $8^{\circ}$ en emballage individuel mais peut être conservé à une température inférieure à $30^{\circ}$ jusqu'à 14 jours.

\section{ADMINISTRATION}

Il faut bien respecter le mode d'emploi et la titration doit toujours être faite au cabinet $d u$ médecin en présence de celui-ci.

Plusieurs considérations pratiques sont indispensables :

1) Uriner avant l'administration du produit.

2) Tenir le pénis le plus allongé possible et vers le haut de façon à ce qu'il soit droit au moment de l'introduction de la canule dans son axe.

3) Après l'administration du MUSE, il faut masser le pénis en le roulant entre deux mains vers le haut pendant 10 secondes et rester en position debout ou mieux en marchant pendant 10 à 15 minutes. On peut s'aider de sérum physiologique stérile à mettre sur la canule pour faciliter son introduction.

\section{RESULTATS DE L'ETUDE EUROPEN- NE ET AMERICAINE A UN AN}

L'étude européenne a porté sur 249 patients qui ont été testés au cabinet, obtenant $64 \%$ de succès.

A trois mois, 159 patients ont continué l'étude avec $74 \%$ de succès et enfin à un an, 106 patients ont complété l'étude avec $55 \%$ de succès ce qui correspond à un an à $35,2 \%$ des patients entrés dans l'étude qui continuent le traitement et qui sont satisfaits $[16,17]$ (tableau 1). 
Dans l'étude US [11], 1511 patients ont été traités au cabinet avec $66 \%$ de succès et à trois mois, 996 patients avec $64,9 \%$ de succès, ce qui donne à un an environ $40 \%$ des patients inclus dans l'étude US, utilisant le MUSE avec succès et $72 \%$ des applications du système sont efficaces.

La tolérance du MUSE qui a été étudiée sur l'ensemble des études, c'est à dire 2591 patients [7].

968 patients ont complété l'étude à 6 mois.

581 l'étude à 12 mois.

211 l'étude à 18 mois.

90 l'étude à 24 mois.

\section{On obtient :}

$29 \%$ douleurs péniennes modérées.

$5 \%$ urétrorragies minimes.

$2 \%$ vertiges

$1,4 \%$ fibrose.

$<0,1 \%$ priapisme

$0 \%$ rétrécissement urétral.

$1,4 \%$ infection urinaire.

\section{ETUDE CRITIQUE DES RESULTATS DE LA LITTERATURE}

Plusieurs séries ont été publiées avec des résultats variables :
1. L'étude de la Mayo-Clinic sur 442 patients par les Docteurs GETTMAN et coll. [5].

Les patients ont été répartis comme suit :

$35 \%$ après prostatectomie radicale.

$33 \%$ d'étiologie artérielle.

$10 \%$ de diabétiques.

Les résultats obtenus sont donc de $51 \%$ de réponses positives avec $42 \%$ de patients qui continuent le traitement à domicile (avec la dose de $1000 \mathrm{mcg}$ ). Les effets secondaires à domicile sont de $57,5 \%$ de brûlures urétrales, ce qui conduit $10 \%$ des patients à abandonner le système à cause des effets secondaires. On obtient donc $38 \%$ de patients satisfaits interrogés par questionnaire. Il faut noter que les douleurs urétrales les plus minimes ont été colligées par les auteurs ce qui explique le chiffre important.

\section{WERTHMAN ET COLL [15]}

Sur 100 patients qui avaient été auparavant traités en injections intra-caverneuses pour leur dysfonction érectile, on obtient $7 \%$ d'érection rigide $5 / 5$ avec le MUSE et $30 \%$ d'érections $4 / 5$, soit $37 \%$ de succès contre respectivement $49 \%$ d'érections $5 / 5$ et $40 \%$ d'érections $4 / 5$ avec les injections intra-caverneuses, soit $89 \%$ de succès, ce qui montre la grande différence d'efficacité.

\section{Tableau 1 : Etude européenne}

\begin{tabular}{lccc}
\hline & AU CABINET & 3 MOIS & 1 AN \\
\hline $\begin{array}{l}\text { NOMBRE DE } \\
\text { PATIENTS }\end{array}$ & 249 & 159 & 106 \\
$\begin{array}{l}\text { Traitement } \\
\text { complété } \\
\text { avec succès (\%) }\end{array}$ & $64 \%$ & $74 \%$ & $55 \%$ \\
$\begin{array}{l}\text { Traitement interrompu : } \\
\begin{array}{l}\text { Effets secondaires } \\
\text { Absence d'efficacité }\end{array}\end{array}$ & $4 \%$ & $3 \%$ & $8 \%$ \\
\hline
\end{tabular}




\section{FULGHAM et coll [3]}

Sur 115 patients, obtient $27 \%$ de patients ayant une rigidité suffisante pour la pénétration.

\section{GALEA et coll.}

Sur 107 patients avec 3 mois de follow up, avec le système MUSE.

Les résultats sont les suivants :

$6,5 \%$ d'érection $5 / 5$

$72 \%$ érection partielle

$20 \%$ d'échecs.

$\mathrm{Au}$ bout de trois mois, seuls $24 \%$ des patients sont satisfaits du système MUSE.

\section{PORST et coll [13]}

103 patients sont traités avec MUSE :

Erections 5/5:10\%

Erections $4 / 5: 33 \%$,

Soit $43 \%$ de succès.

\section{LES POSSIBILITES D'AMELIORA- TION DU SYSTEME MUSE}

Deux voies de recherches sont en cours.

1) L'adjonction d'un alpha-bloquant qui est la PRAZOZIN associée à l'ALPROSTADIL : Il s'agit du système ALIBRA.

2) L'utilisation d'un garrot élastique l'ACTIS

\section{Le système ALIBRA.}

Les résultats de l'étude du groupe ALPROSTADIL-PRAZOZIN-STUDY GROUP [13], sur 394 patients qui ont reçu une titration de MUSE seul ou d'ALIBRA en comparaison de 125 à $1000 \mathrm{mcg}$ d'ALPROSTADIL et de 250 à $1000 \mathrm{mcg}$ de PRAZOZIN. Les résultats donnent $70 \%$ d'érection $4 / 5$ ou $5 / 5$ en résultats globaux, le système ALIBRA étant un succès chez $23 \%$ des patients qui avait été un échec avec le système MUSE seul (89 / 394 patients).

\section{Le système ACTIS}

Lewis et Coll [9] ont étudié 160 patients qui utilisent l'ACTIS pendant 10 minutes après l'application du MUSE. Il s'agit d'un petit garrot élastique très original, une sorte de lasso que le patient peut mettre très facilement à la racine de la verge.

On obtient alors avec ce système $64 \%$ de succès globaux avec le MUSE contre $43 \%$ qui avaient été obtenus dans l'étude US avec le MUSE seul. Donc amélioration des résultats selon les investigateurs.

\section{COMPARAISON INJECTIONS INTRA-CAVERNEUSE ET MUSE}

Plusieurs études comparatives ont été publiées dans la littérature.

\section{PORST et coll [12]}

103 patients ont été étudiés, obtenant $43 \%$ de réponses positives au MUSE, contre $70 \%$ de réponses positives aux injections intra-caverneuses de prostaglandine E.1.

Il existe surtout ce qui est notable une différence de la qualité de l'érection. En effet, on obtient $10 \%$ d'érections rigides $5 / 5$ avec MUSE contre $48 \%$ avec les injections intra-caverneuses et $33 \%$ d'érections $4 / 5$ avec le MUSE contre $22 \%$ d'érections $4 / 5$ avec l'injection intra-caverneuse de prostaglandine E.1.

Indéniablement l'efficacité des injections intracaverneuses de PGE 1 est meilleure et de plus la rigidité est de meilleure qualité.

\section{GHAZI et colls [4]}

font tester les injections intra-caverneuses et le MUSE à 125 patients pendant 15 jours à domicile. Les résultats sont là aussi parlants avec $79 \%$ de succès pour les injections intracaverneuses de PGE 1 contre $48 \%$ pour le MUSE.

Mais il faut noter néanmoins que $89 \%$ des patients considèrent l'application du MUSE beaucoup plus facile que les injections intracaverneuses.

3. Une étude de la littérature par métaanalyse par FLYNN et coll [2] a retrouvé 70 $\%$ d'érections suffisantes pour la pénétration en résultat global pour les injections intracaverneuses, contre $45 \%$ pour le MUSE. 
Les effets secondaires étant en moyenne de $20 \%$ de douleurs pour le MUSE et les injections intra-caverneuses avec en plus $5 \%$ d'urétrorragies pour le MUSE.

4. Enfin l'étude de MONTORSI et coll est intéressante pour exprimer la meilleure acceptabilité du MUSE par rapport aux injections intra-caverneuses et qui est somme toute un avantage indéniable du MUSE.

50 patients visionnent une cassette vidéo montrant l'injection intra-caverneuse de PGE 1 avec auto-injecteur et l'insertion du MUSE.

$70 \%$ trouvent le MUSE moins invasif que les injections intra-caverneuses.

$20 \%$ trouvent que cela est équivalent.

$10 \%$ trouvent que le MUSE est plus invasif que les injections intra-caverneuses.

Ceci bien sûr ne remet nullement en cause la meilleurs efficacité des injections intra-caverneuses puisque les résultats donnés dans cette étude par MONTORSI sont les suivants :

$40 \%$ des patients ont une érection $5 / 5$ avec le MUSE contre $80 \%$ avec les injections intracaverneuses.
En résumé on peut dans le tableau cidessous colliger les avantages et les inconvénients du MUSE et des injections intra-caverneuses (tableau 2).

\section{COMPARAISON MUSE ET SILDENAFIL}

La seule étude très documentée a été rapportée par STIEF et coll [14] au congrès mondial d'Amsterdam en 1998.

46 patients ont été inclus avec 3 critères :

1) Pas d'étiologie psychogène.

2) Un test intra-caverneux à la PGE 1 positif de $2,5 \mathrm{mcg}$ à $20 \mathrm{mcg}$.

3) Pas de contre indication au MUSE et au SILDENAFIL (VIAGRA), ce qui introduit bien sûr un biais.

Les patients sont testés par injections intracaverneuses d'abord par un test, puis une titration est faite pour le MUSE et le VIAGRA et les patients vont utiliser successivement ensuite le MUSE et le VIAGRA à domicile avec un dosage adapté.

$24 \%$ ont une réponse positive au MUSE et au SILDENAFIL.

Tableau 2 : Comparaison entre MUSE et IIC de PGE 1

\begin{tabular}{lcc}
\hline & MUSE & IIC (PGE 1) \\
ACCEPTABILITE & MEILLEURE & MOINS BONNE \\
PRESENTATION & PLUS SIMPLE & PLUS COMPLIQUEE \\
SUCCES (\%) & $30 \%$ à 50\% & $65 \%$ à $85 \%$ \\
SUCCES EN CAS & ENVIRON 50\% & \\
DE PROSTATECTOMIE & & ENVIRON $70 \%$ \\
RADICALE (\%) & 35 à 40\% à un an & $50 \%$ à un an \\
ABANDON & $<1 \%$ & $6-7 \%$ (PGE1) \\
FIBROSE \% & $<0,5 \%$ & $<2 \%$ \\
ERECTION PROLONGEE & BRULURES URETRALES & DOULEURS PENIENNES \\
TOLERANCE & ENVIRON 20-30 \% & $35 \%$ DOULEURS \\
\hline
\end{tabular}


$48 \%$ ont une réponse positive au SILDENAFIL, et négative au MUSE..

$15 \%$ ont une réponse positive au MUSE et négative au VIAGRA.

$13 \%$ ont une réponse négative au MUSE et au VIAGRA.

$\mathrm{Au}$ total la réponse positive avec succès pour le MUSE est de $39 \%$, contre $72 \%$ au SILDENAFIL.

En résumé, le tableau ci-dessous reprend les différents avantages et inconvénients du MUSE et du SILDENAFIL (tableau 3).

\section{QUELLES INDICATIONS VA-T-IL RESTER POUR PROPOSER LE MUSE DANS LE TRAITEMENT DE L'INSUFFI- SANCE ERECTILE.}

Le MUSE devrait être commercialisé en France aux alentours de février 1999 et on peut ainsi résumer les possibles indications de ce traitement dans l'insuffisance érectile.

1) Les contres indications connues et formelles du Sildenafil.

2) Les populations à haut risque (coronariens ou diabétiques) où le choix du patient et du clinicien ne se portera pas sur le VIAGRA.

3) Les patients plus âgés qui refusent les injections intra-caverneuses préférant se contenter d'érections moins rigides.

4) Les prostatectomisés où l'on pourrait utiliser le MUSE en post-opératoire immédiat en balance avec les injections intra-caverneuses.

5) Les patients qui abandonnent provisoirement ou définitivement les injections intracaverneuses en raison de la survenue de fibrose due à ces injections et qui ne peuvent pas bénéficier du Sildenafil.

6) Les maladies de La Peyronie peu invalidante après échec du Sildenafil.

7) Les très rares cas de " sauvetage " de prothèses insuffisamment efficaces ou explantées en utilisant le MUSE seul ou en association à un autre système par exemple le VACUUM.

8) Les cas où l'on recherche une turgescence du gland.

Tableau 3 : Comparaison entre MUSE et Sildénatif

\begin{tabular}{lcc}
\hline & MUSE & VIAGRA \\
\hline ACCEPTABILITE & MOINS BONNE & MEILLEURE ++ \\
PRESENTATION & MOINS BONNE & MEILLEURE ++ \\
DELAI D'ACTION & 15 à 30 minutes ++ & $1 \mathrm{~h}$ à $3 \mathrm{~h}$ \\
SUCCES \% & 30 à $60 \%$ & $40 \%$ à $80 \%$ \\
SUCCES EN CAS DE PR $(\%)$ & environ $50 \%$ & $<40 \%$ \\
ABANDON \% & $35 \%$ en un an & FAIBLE \\
FIBROSE \% & $<1 \%$ & 0 ? \\
ERECTION PROLONGEE & $<0,5 \%$ & $<, 1 \%$ \\
TOLERANCE & BRULURES URETRALES & CEPHALEES : 15 \% \\
& ENVIRON 35 \% & BOUFFEES CHALEUR : $10 \%$ \\
CONTRE INDICATION & STENOSE DU MEAT & PRISE DE DERIVES NITRES \\
& URETRAL ASS. & \\
& MEDICAMENTEUSE & Pb DES CORONARIENS ? ?
\end{tabular}




\section{CONCLUSION}

Le système MUSE est une alternative efficace dans le traitement de la dysfonction érectile, il garde une place dans l'arsenal thérapeutique du clinicien avec environ 35 à $40 \%$ de succès attendu. Il est évident que sa place s'est trouvée réduite depuis l'apparition du Sildenafil qui est et sera première ligne thérapeutique de l'insuffisance érectile avec une très grande efficacité.

Le MUSE demeure nettement moins efficace que les injections intra-caverneuses pour ce qui est des traitements locaux mais son acceptabilité est meilleure.

\section{REFERENCES}

1. ADAIKAN PG, KOTTEGODA SR, RATNAM SS. A possible role for prostaglandin $\mathrm{E}$, in human penile erection. In : Abstract book Second World Meeting on Impotence, Prague 1986, Abs. 2.6.

2. FLYNN T.N, GUEST J.F. Intra corporeal and transurethral. Application of alprostadil : A review of litterature. Int. J.Impot. Res.1998, 10, Supplt 3, S 47.

3. FULGHAM P.F, COCHRAN J.S, DENMAN J.L and al : Disappointing initial results with transurethral alprostadil for erectile dysfunction in an urology practice setting. J.Urol.1998 160,6, 2041 - 6.

4. GHAZI S.A, ALMELIGY A. Transurethral alprostadil and the prostaglandin E1 intra-corporal injection in the treatment of erectile dysfunction : A comparative study. Int.J.Impot. Res.1998, 10, Supplt 3, S 13.

5. GETTMAN M.T, NIENOW D.A, PATTERSON D.E and al : Muse therapy for male sexual dysfunction. In 454 consecutive patients : subjective and objective results at initial evaluation at dose titration. Int $\mathbf{J}$. Impot. Res. 1998,10 (Supplt3), S 552.

6. HELLSTROM W.J.G, SIKKA S.C, WANG R and al : Does transurethral alprostadil affect the concentration of prostaglandins in the human ejaculate or the viability and motility of human sperm ? Int. J Androl.1997, 20(suppl1) : 104.

7. ISHII N, WATANABE H, IRISAWA C, KIKUCHI Y. Therapeutic trial with prostaglandin $\mathbf{E 1}$ for organic impotence. In : Abstract Book Second World Meeting on Impotence , Prague 1986, Abs. 11.2

8. LABASKY R.C, SPIVACK A.P and al : Transurethral alprostadil for the treatment of erectile dysfunction : Two-year safety update. J. Urol. 1998, 159,5 (supplt) $-235$

9. LEWIS RW, WELDON K, NEMO K. Combined use of transurethral alprostadil and adjustable penile constriction band in men with erectile dysfunction , results from a multicenter trial. Int.J.Impot. Res.1998,10, supplt 3, S49.

10. PADMA-NATHAN H ET AL. Hemodynamic effects of intraurethral alprostadil : The medicated Urethral System for Erection (MUSE*). J Urol 1994, 151 :345 A : 469.

11. PADMA-NATHAN H., HELLSTROM W.J.G, KAISER F.E and al : Treatment of men with erectile dysfunction with transurethral alprostadil. NewEngl. J.Med $1997: 336: 1-7$.

12. PETERSON C.A, BENNETT A.H, HELLSTROM W.J.G and al. Erectile response to transurethral alprostadil, prazosin and alprostadil-prazosin combinations. J.Urol 1998, 159, 1523-1528.

13. PORST H. Transurethral alprostadil with Muse V.S intra-cavernous alprostadil. A comparative study in 100 patients with erectile dysfunction. Int. J.Impot. Res.1997,9,187-192.

14. STIEF C.G, MESCHI M., SCHULTHEISS D. Efficacy and acceptance of intra-cavernous versus intra-urethral PGE1 versus sildenafil. Int. J.Impot.Res.1998, 10, Supplt 3, S 46.

15. WERTHMAN P., RAJFER J. Muse therapy : Preliminary clinical observations. Urology 1997, 50 (5) : 809-11

16. WILLIAMS G, ABBOU C.C, AMAR E.T and al : Efficacy and safety of transurethral alprostadil therapy in men with erectile dysfunction. Brit J.Urol 1998,81,889-894.

17. WILLIAMS G, ABBOU C.C, AMAR E.T and al : The effect of transurethral alprostadil on the quality of life of men with erectile dysfuncetion and their partners. Brit. J. Urol 1998, 82, 847-854.

\section{ABSTRACT}

What is the place left for transurethral alprostadil in the treatment of erectile dysfunction?

E. Amar

Transurethral alprostadil (Muse) is an effective and safe treatment of erectile dysfunction. Nevertheless, the result after its exit on the US market two years ago were not as good as the investigational studies which claimed aroud $60 \%$ of scess rate. In the litterature success rate were between $35 \%$ and $40 \%$ all together. The Muse had a better acceptance than the intra-cavernous injection despite a lower success rate (40 \% v.s $75 \%)$. Since Sildenafil came on the market, it seems that 
the place of Muse is reduced because comparative studies give better results for Sildenafil than Muse (70 \% v.s $40 \%$ ) and of course with a better acceptance. Nevertheless there are absolute and relative counter-indications to the Sildenafil which could benefit to the treatment by Muse.

All the comparative studies, IIC, v.s Muse and sildenafil v.s Muse will be studied in this article.

In conclusion Muse should be used as an alternative and should remain an effective tool that must be available to all physicians dealing with erectile dysfunction, perhaps using new formula with a combination of alprostadil with an alpha-blocker.

Key-Words : Erectile dysfunction - transurethral alprostadil-intra-cavernous injection - Sildenafilcomparative study-Prostaglandine E1. 